\title{
Diastematomyelia: A Surgical Case with Long-Term Follow-Up
}

\author{
Hirofumi Bekki ${ }^{1}$, Yuichiro Morishita ${ }^{2}$, Osamu Kawano ${ }^{2}$, Keiichiro Shiba ${ }^{2}$, Yukihide Iwamoto ${ }^{1}$ \\ ${ }^{1}$ Department of Orthopedic Surgery, Kyushu University, Fukuoka, Japan \\ ${ }^{2}$ Department of Orthopedic Surgery, Spinal Injuries Center, Iizuka, Japan
}

Few reports have described the involvement of syringomyelia associated with diastematomyelia in the etiology of neurological deficits. We reported a case with syringomyelia associated with diastematomyelia. A female patient with diastematomyelia was followed up clinically over 14 years. At the age of 8 , she developed clubfoot deformity with neurological deterioration. Motor function of the right peroneus demonstrated grade 2 in manual muscle tests. Continuous intracanial bony septum and double cords with independent double dura were observed at upper thoracic spine. Magnetic resonance imaging revealed a tethering of the spinal cord and syringomyelia distal to the level of diastematomyelia. Extirpation of the osseum septum and duralplasty were performed surgically. She grew without neurological deterioration during 7 years postoperatively. A long-term followed up case with syringomyelia that was possibly secondary to the tethering of the spinal cord associated with diastematomyelia, and effective treatment with extirpation of the osseum septum and duralplasty was described.

Keywords: Diastematolyelia; Syringomyelia; Tethered cord syndrome

\section{Introduction}

Split cord malformations (SCMs) are those in which the spinal cord is divided along a portion of its length to form a double tube. They are rare congenital spinal disorders. Two distinct forms, diastematomyelia and diplomyelia, have been described. SCM is divided into two types: type I, 2 hemicords in double dura and with bony or cartilaginous septum; type II, 2 hemicords in a single dura without a septum [1]. The location of the lesion can occur at any spinal level but is most frequent in the lower thoracic or upper lumbar areas $[2,3]$.

Clinically, SCM occurs predominantly in females [2]. The majority of patients have midline thoracic or lumbar cutaneous abnormalities associated with spinal dysra- phism including hairy patches, dimples, hemangiomas, lipomas, and sinus tracts [3]. Sixty to seventy percent of patients with diastematomyelia have congenital scoliosis [3], and usually a neurologic abnormality results from the dysfunction of the distal spinal cord and conus medullaris. Neurological deterioration was considered to be caused by the tethering of the spinal cord secondary to the growth of the spinal canal [4]. The accurate etiology of neurological deficits, however, is still the matter at discussion.

In our case, the patient also demonstrated the tethering of the spinal cord associated with diastematomyelia, and developed syringomyelia distal to the level of diastematomyelia. To the best of our knowledge, few reports have, thus far, described the participation of syringomyelia associated with diastematomyelia in the etiology of neuro- 
logical deficits. We hereby report a surgical case with 14 years of long-term follow up demonstrating the association of syringomyelia with diastematomyelia.

\section{Case Report}

A female patient with diastematomyelia was clinically followed for over 14 years. She had been in normal delivery, but indicated the skin stigma on her back at newborn checkup. At age one, she was diagnosed with diastematomyelia based on the radiological examination at our facility. Plain radiographs showed spina bifida, widening of the interpedicular distance at upper thoracic spine, and congenital mild single thoracic scoliosis (T8-L3 Cobb angle: $10^{\circ}$ ) (Fig. 1). She was followed yearly at our facility.

At age 8, a hairy patch appeared on her back, and she demonstrated limping due to clubfoot deformity with neurological deterioration. The patient was decided to be treated surgically.

\section{Neurological examination}

Sensory at lower extremity was intact, but, motor at right peroneus demonstrated 2 in manual muscle testing. Moreover, hyperreflexia in right patella tendon and positive right Babinski reflex were observed.

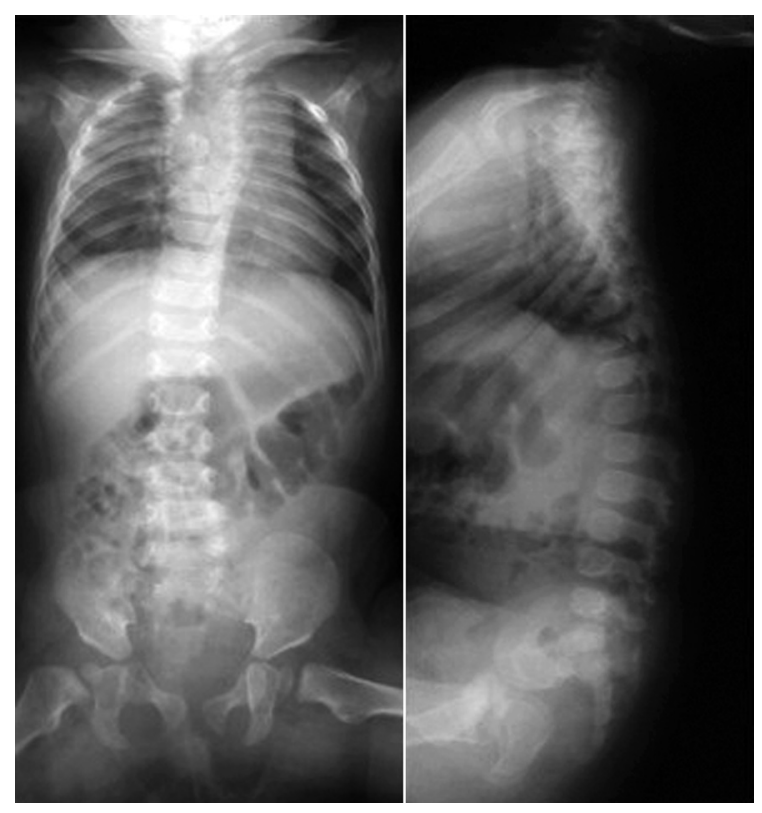

Fig. 1. Plain radiographs demonstrated a congenital mild single thoracic scoliosis. The irregular images of vertebra columns and extension of intrapedicle distance were seen at upper to middle thoracic region.

\section{Radiological examination}

There was no evident deterioration of congenital scoliosis during the 7 year follow up period in plain radiographs. Continuous intracanal bony septum at the level of T2/3 to $\mathrm{T} 5$, double cords with independent double dura at the level of T2 to T6, and split cord at the level of T1-T11 were observed in postmyelogram computed tomography (Fig. 2). In magnetic resonance imaging (MRI), the sy-
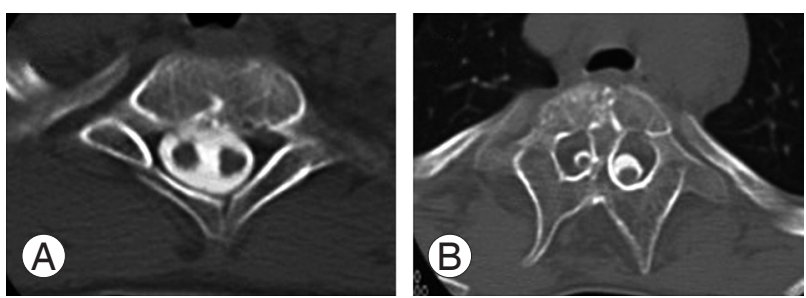

Fig. 2. (A) Split cord at the level of T1. (B) Continuous intracanal bony septum and double cords with independent double dura at the T4 level.
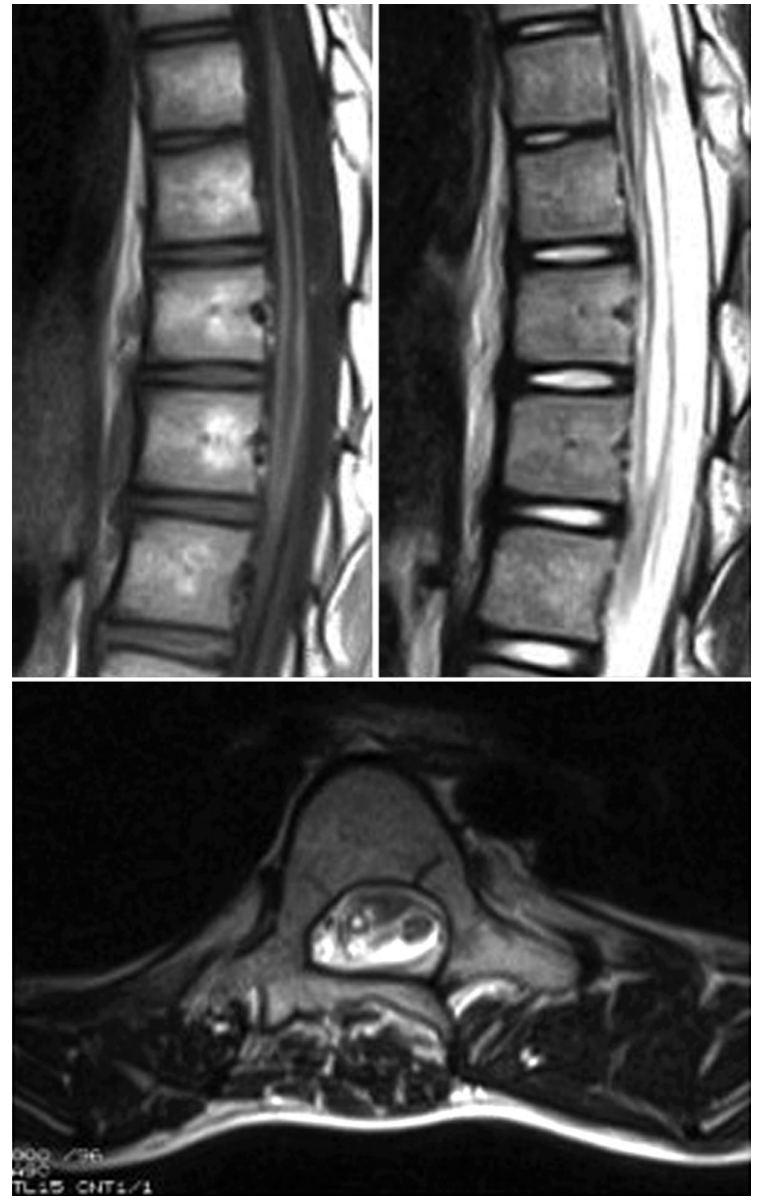

Fig. 3. Magnetic resonance imaging demonstrated syringomyelia on the right sprit cord at the level of T8 to T11. 
ringomyelia on right split cord at the level of T8 to T11, distal to the level of diastematomyelia was observed (Fig. $3)$.

\section{Surgical treatment}

The osseus anomaly, petaloid spinous processes at the level of T3 to T9 and right median intracanal bony septum at the level of T2/3 to T5, and type I SCM were observed intraoperatively (Fig. 4).

T2-6 laminectomy was performed. Tethering of the split spinal cords were observed, and dural pulsation was seen only at normal part of the dural sac, not at the double duras. After resection of the intracanal osseum septum, the tension of the split cords was released. Duralplasty with the bifid dural sacs converted to a single sac were performed surgically.

Postoperative MRI showed gradual shrinkage of syringomyelia (Fig. 5), and the patient grew without a neurological deterioration during seven postoperative years. Moreover, surgical treatment did not affect the progression of congenital scoliosis (Fig. 6).
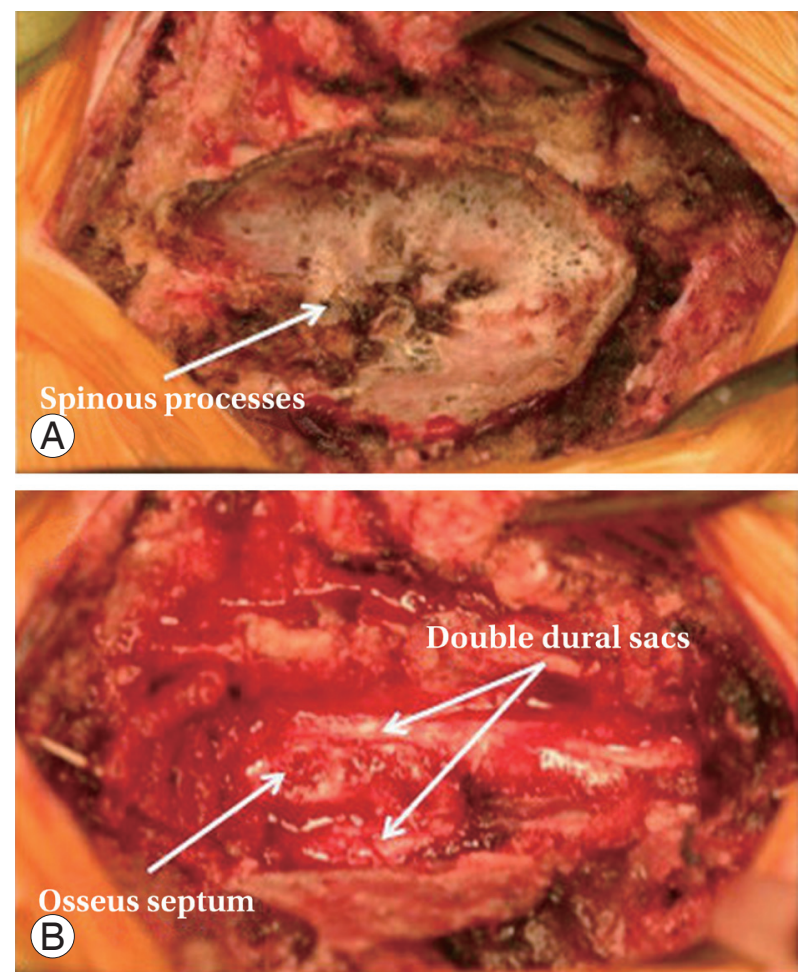

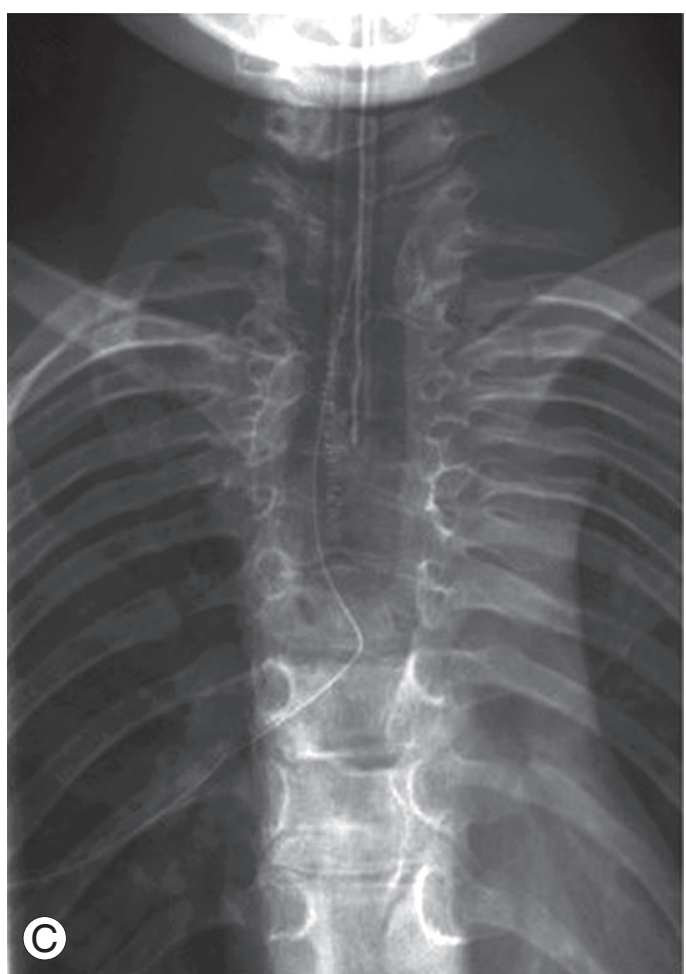

Fig. 4. (A) Before laminectomy; petaloid spinous processes were observed. (B) After laminectomy; intracanal osseus septum and independent double duras were observed. (C) Postoperative plain radiograph.
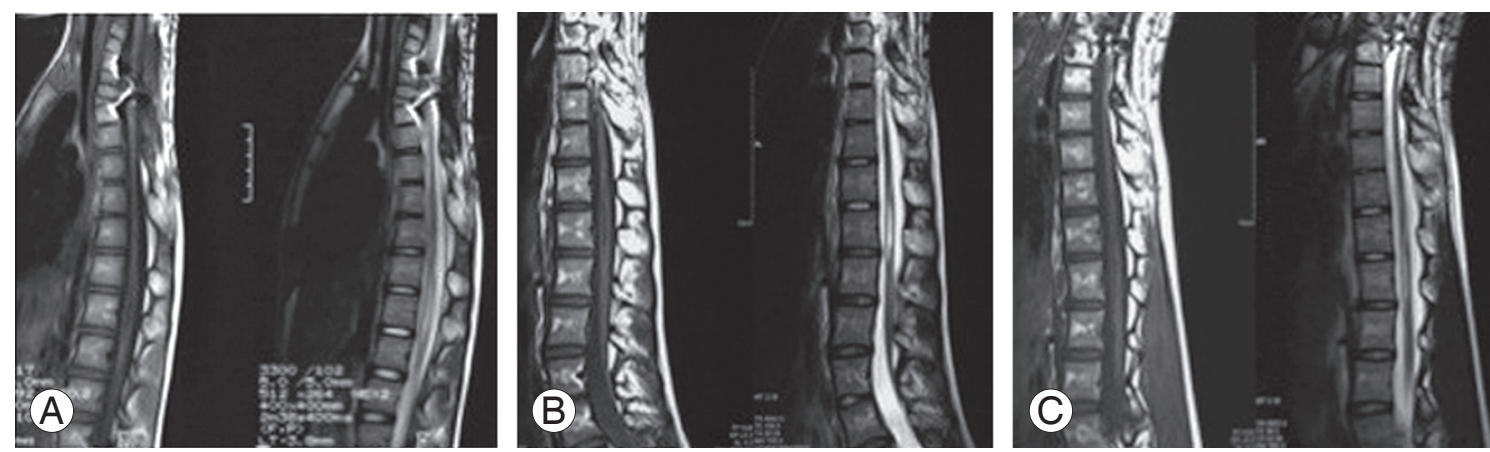

Fig. 5. Postoperative magnetic resonance imaging showed gradual shrinkage of syringomyelia. (A) Two years postoperatively. (B) Four years postoperatively. (C) Six years postoperatively. 


\section{Discussion}

Progressive neurological deterioration in patients who have diastematomyelia has been attributed to the damage of the spinal cord resulting from either tethering or traction [5]. The degree of tethering of the cord may be related to the age of the patients, and the risk of developing neurological deficits increases with age [4].

In our case, the patient also demonstrated tethering of the spinal cord secondary to the growth of the spinal canal, and the neurological deficits had developed at relatively older age. We hypothesized that the syringomyelia distal to the level of diastematomyelia might have developed secondary to the tethering of the spinal cord associated with diastematomyelia. Milhorat (TH Milhorat, personal communication, 1997) suggested that the terminal cavitation represents a noncommunicating syrinx process caused by the coexisting mechanisms of tethering induced cord ischemia cerebrospinal fluid pulse pressure gradients resulting from the reduced compliance of the spinal subarachnoid space due to the pathological tether-
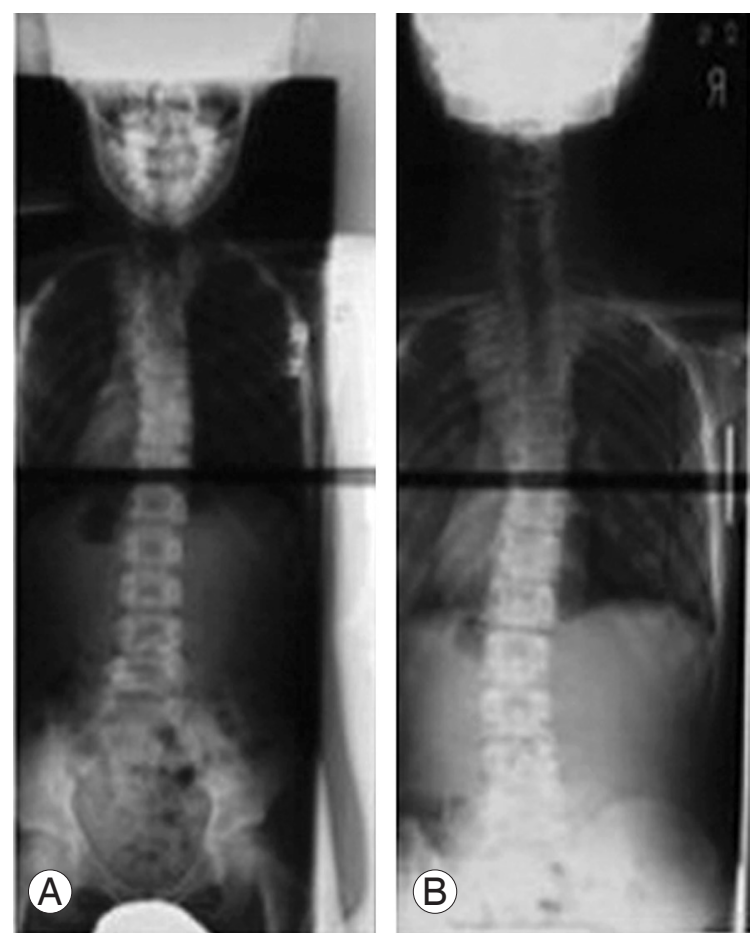

Fig. 6. Plain-standing radiographs; surgical treatment did not affect significant progression of congenital scoliosis. (A) At the time of surgery. (B) Six years postoperatively. ing conditions. The damages to the spinal cord may lead local injury to the neural elements, and in turn result in neurological deficits. We could not conclude the efficacy of the duralplasty. However, we hypothesized that the intracanal osseum resection might prevent from further tethering of the spinal cord following bony growth, and duralplasty might obtain the uniformity of cerebrospinal fluid circulation and might lead to the gradual shrinkage of syringomyelia.

The management of SCM is one of the most controversial issues in the treatment of spinal congenital malformations. In our case, the patient did not demonstrate complete neurologic recovery postoperatively. We regretted that we should have performed surgical treatment before the appearance of neurological deficits. The necessity of duralplasty was the matter at hand, but, the patient received satisfied results without a neurological deterioration or a progression of congenital scoliosis during 7 years after the surgery.

\section{Conflict of Interest}

No potential conflict of interest relevant to this article was reported.

\section{References}

1. Pang D, Dias MS, Ahab-Barmada M. Split cord malformation: Part I: A unified theory of embryogenesis for double spinal cord malformations. Neurosurgery 1992;31:451-80.

2. Ersahin Y, Mutluer S, Kocaman S, Demirtas E. Split spinal cord malformations in children. J Neurosurg 1998;88:57-65.

3. Hood RW, Riseborough EJ, Nehme AM, Micheli LJ, Strand RD, Neuhauser EB. Diastematomyelia and structural spinal deformities. J Bone Joint Surg Am 1980;62:520-8.

4. Miller A, Guille JT, Bowen JR. Evaluation and treatment of diastematomyelia. J Bone Joint Surg Am 1993;75:1308-17.

5. Hensinger RN, MacEwen GD. Congenital anomalies of the spine. In: Rothman RH, Simeone FA, editors. The spine. Philadelpha: Saunders; 1982. p.255-63. 